\title{
A Machine-Learning Based Load Prediction Approach for Distributed Service-Oriented Applications
}

\author{
Jun Wang, Yi Ren, Di Zheng, and Quan-Yuan Wu \\ School of Computer Science, \\ National University of Defence Technology, \\ Changsha, Hunan, China 410073 \\ junwang@nudt.edu.cn
}

\begin{abstract}
By using middleware, we can satisfy the urgent demands of performance, scalability and availability in current distributed service-oriented applications. However to the complex applications, the load peak may make the system suffer extremely high load and the response time may be decreased for this kind of fluctuate. Therefore, to utilize the services effectively especially when the workloads fluctuate frequently, we should make the system react to the load fluctuate gradually and predictably. Many existing load balancing middleware use the dampening technology to make the load to be predicative. However, distributed systems are inherently difficult to manage and the dampening factor cannot be treated as static and fixed. The dampening factor should be adjusted dynamically according to different load fluctuate. So we have proposed a new technique based on machine learning for adaptive and flexible load prediction mechanism based on our load balancing middleware.
\end{abstract}

Keywords: Service-Oriented Applications, Load Prediction, MachineLearning, Middleware.

\section{Introduction}

To service many increasing online clients those transmit a large, often busty, number of requests and provide dependable services with high quality constantly, we must make the distributed computing systems more scalable and dependable. Effective load balancing mechanisms must be made use of to distribute the client workload equitably among back-end servers to improve overall responsiveness. Load balancing mechanisms can be provided in any or all of the following layers:

- Network-based load balancing: This type of load balancing is provided by IP routers and domain name servers (DNS). Web sites often use network-based load balancing at the network layer (layer 3 ) and the transport layer (layer 4).

- OS-based load balancing: This type of load balancing is provided by distributed operating systems via load sharing, and process migration [1] mechanisms.

- Middleware-based load balancing: This type of load balancing is performed in middleware, often on a per-session or a per-request basis. The key enterprise 
applications of the moment such as astronavigation, telecommunication, and finance all make use of the middleware based distributed software systems to handle complex distributed applications.

There are different realizations of load balancing middleware. For example, stateless distributed applications usually balance the workload with the help of naming service [2]. But this scheme of load balancing just support static non-adaptive load balancing and can't meet the need of complex distributed applications. For more complex applications, the adaptive load balancing schema $[3,4,5]$ is needed to take into account the load condition dynamically and avoid override in some node. Many existing load balancing middleware use the dampening technology to make the load to be predicative. However, distributed systems are inherently difficult to manage and the dampening factor cannot be treated as static and fixed. So, in this paper we design and implement an efficient load prediction method for service-based applications. Clients can learn the best policy by employing reinforcement based learning technique. Reinforcement based learning is an un-supervised learning, in which agents learn through trial and error interactions with environment.

\section{Load Prediction Method for Service-Oriented Applications}

\subsection{Model of the Load Balancing Middleware}

Our middleware provides load balancing for the service-oriented applications, prevents bottlenecks at the application tier, balances the workload among the different services and enables replication of the service components in a scalable way. The core components are as follows:

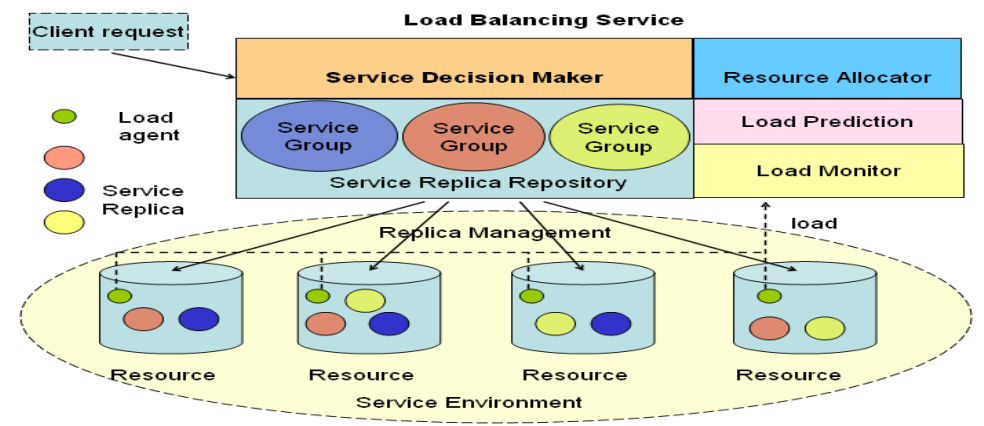

Fig. 1. Components of the Load Balancing Middleware

Service Replica Repository: Instances of services need to register with the Service Group. All the references of the groups are stored in the Service Replica Repository. A service group may include several replicas and we can add or remove replicas to the groups. The main purpose of the service group is to provide a view containing simple information about the references to the locations of all replicas registered with group. The uses need not to know where the replica is located. 
Service Decision Maker: This component assigns the best replica in the group to service the request based on the algorithms configured in our load balancing policy [6]. The service decision maker acts as a proxy between the client and the dynamic services. It enables transparency between them without letting the client knowing about the multiple distributed service replicas.

Load Monitor: Load monitor collects load information from every load agent within certain time interval. The load information should be refreshed at a suitable interval so that the information provided is not expired.

Load Agent: The purpose of load agent is to provide load information of the hosts it resides when requested by load monitor. As different services might have replicas in the same host, it is hard to presume the percentage of resource is being used by which service at particular moment. Therefore, a general metric is needed to indicate the level of available resources at the machine during particular moment.

Resource Allocator: The purpose of this component is to dynamically adjust the resource to achieve a balance load distribution among different services. In fact, we control the resource allocation by managing the replicas of different services. For example, this component makes decisions on some mechanisms such as service replication, services coordination, dynamic adjustment and requests prediction.

\subsection{Machine-Learning Based Load Prediction}

As figure 2 shown, the host will suffer extreme loads at time T1 and T2, and if we computing the load basing on the numbers sampling from these two points we will make wrong load balancing decisions. A useful method is Using control theory technology called dampening, where the system minimizes unpredictable behavior by reacting slowly to changes and waiting for definite trends to minimize over-control decisions. The load will be computed in this way:

$$
\text { new_load }=\text { multiplier } * \text { old_load }+(1-m \text { ultiplier }) * n e w \_ \text {load }
$$

The parameter multiplier is the dampening factor and the value of the multiplier is between 0 and 1.The parameter old_load represents for the former load and the new_load represents for the new sampling load. However, distributed systems are inherently difficult to manage and the dampening factor cannot be treated as static and fixed. The dampening factor should be adjusted dynamically according to different load fluctuate. Therefore, we use the machine-learning based load prediction method where the system minimizes unpredictable behavior by reacting slowly to changes and waiting for definite trends to minimize over-control decisions. The simple

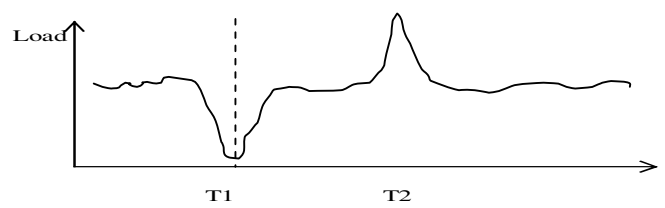

Fig. 2. Affection of the Peak Load 
exponential smoothing method is based on a weighted average of current and past observations, with most weight to the current observation and declining weights to past observations. The formula for exponential moving average is given by equation (2): Where $0 \leq \theta \leq 1$ is know as dynamic dampening factor, $L_{n}$ is the most recent load, $\varphi_{n}$ stores the past history and $\varphi_{n+1}$ is the predicted value of load.

$$
\varphi_{n+1}=\theta L_{n}+(1-\theta) \varphi_{n}
$$

We maintained two exponentially weighted moving averages with different dynamic dampening factors. A slow moving average $(\theta \rightarrow 0)$ is used to produce a smooth, stable estimate. A fast moving average $(\theta \rightarrow 1)$ adapts quickly to changes in work load. The maximum of these two values are used as an account for current load on the service provider.

\section{Conclusions}

To utilize the services effectively especially when the workloads fluctuate frequently, we should make the system react to the load fluctuate gradually and predictably. Distributed systems are inherently difficult to manage and the dampening factor cannot be treated as static and fixed. The dampening factor should be adjusted dynamically according to different load fluctuate. So we have proposed and implemented a new technique based on machine learning for adaptive and flexible load prediction mechanism based on our load balancing middleware.

\section{Acknowledgements}

This work was funded by the National Grand Fundamental Research 973 Program of China under Grant No.2005cb321804 and the National Natural Science Foundation of China under Grant No.60603063.

\section{References}

1. Rajkumar, B.: High Performance Cluster Computing Architecture and Systems, ISBN7.5053-6770-6.2001

2. IONA Technologies, "Orbix 2000." www.iona-iportal.com/suite/orbix2000.htm.

3. Othman, O'Ryan, C., Schmidt, D. C.: The Design of an Adaptive CORBA Load Balancing Service. IEEE Distributed Systems Online(2001)

4. Othman, O., Schmidt, D. C.: Issues in the design of adaptive middleware load balancing. In: ACM SIGPLAN, ed. Proceedings of the ACM SIGPLAN workshop on Languages, Compilers and Tools for Embedded Systems. New York: ACM Press(2001)205-213

5. Othman, O., O'Ryan, C., Schmidt, D.C.: Strategies for CORBA middleware-based load balancing. IEEE Distributed Systems Online(2001) http://www.computer.org/dsonline

6. Gamma, E., Helm, R., Johnson, R., Vlissides, J.: Design Patterns: Elements of Reusable Object-Oriented Software. Reading: Addison-Wesley(2002)223-325 